Research Article

Open Access

Blanka Baculikova* and Jozef Dzurina

\title{
The fourth order strongly noncanonical operators
}

https://doi.org/10.1515/math-2018-0135

Received May 22, 2018; accepted November 26, 2018

Abstract: It is shown that the strongly noncanonical fourth order operator

$$
\mathcal{L} y=\left(r_{3}(t)\left(r_{2}(t)\left(r_{1}(t) y^{\prime}(t)\right)^{\prime}\right)^{\prime}\right)^{\prime}
$$

can be written in essentially unique canonical form as

$$
\mathcal{L} y=q_{4}(t)\left(q_{3}(t)\left(q_{2}(t)\left(q_{1}(t)\left(q_{0}(t) y(t)\right)^{\prime}\right)^{\prime}\right)^{\prime}\right)^{\prime} .
$$

The canonical representation essentially simplifies examination of the fourth order strongly noncanonical equations

$$
\left(r_{3}(t)\left(r_{2}(t)\left(r_{1}(t) y^{\prime}(t)\right)^{\prime}\right)^{\prime}\right)^{\prime}+p(t) y(\tau(t))=0
$$

Keywords: canonical operator, fourth order differential equations

MSC: $34 \mathrm{~K} 11,34 \mathrm{C} 10$

\section{Introduction}

In the paper, we consider the fourth order delay differential equation

$$
\left(r_{3}(t)\left(r_{2}(t)\left(r_{1}(t) y^{\prime}(t)\right)^{\prime}\right)^{\prime}\right)^{\prime}+p(t) y(\tau(t))=0
$$

where $r_{i} \in C^{(4-i)}\left(t_{0}, \infty\right), r_{i}(t)>0, i=1, \ldots, 3, p>0, \tau(t) \leq t, \tau^{\prime}(t)>0$ and $\tau(t) \rightarrow \infty$ as $t \rightarrow \infty$.

Fourth-order differential equations naturally appear in models concerning physical, biological, and chemical phenomena, such as, for instance, problems of elasticity, deformation of structures, or soil settlement, for example, see [1]. In mechanical and engineering problems, questions concerning the existence of oscillatory solutions play an important role. During the past decades, there has been a constant interest in obtaining sufficient conditions for oscillatory properties of different class of fourth order differential equations with deviating argument, see [1-13].

\footnotetext{
*Corresponding Author: Blanka Baculikova: Department of Mathematics, Faculty of Electrical Engineering and Informatics, Technical University of Košice, Letná 9, 04200 Košice, Slovakia, E-mail: blanka.baculikova@tuke.sk

Jozef Dzurina: Department of Mathematics, Faculty of Electrical Engineering and Informatics, Technical University of Košice, Letná 9, 04200 Košice, Slovakia, E-mail: jozef.dzurina@tuke.sk
} 
As far as the oscillation theory of fourth-order differential equations is concerned, the problem of investigating ways of factoring disconjugated operators

$$
\mathcal{L} y \equiv\left(r_{3}(t)\left(r_{2}(t)\left(r_{1}(t) y^{\prime}(t)\right)^{\prime}\right)^{\prime}\right)^{\prime},
$$

which are crucial in studying the perturbed differential equations such as $(E)$, has been of special interest. Motivated by the famous work of George Polya, Trench [2] showed that if operator $\mathcal{L} y$ is strongly noncanonical, that is,

$$
\int^{\infty} \frac{1}{r_{i}(s)} \mathrm{d} s<\infty, \quad i=1,2,3,
$$

then it can be written in an essentially unique canonical form as

$$
\mathcal{L} y=q_{4}(t)\left(q_{3}(t)\left(q_{2}(t)\left(q_{1}(t)\left(q_{0}(t) y(t)\right)^{\prime}\right)^{\prime}\right)^{\prime}\right)^{\prime},
$$

so that $q_{i} \in C^{(4-i)}\left(t_{0}, \infty\right), q_{i}(t)>0, i=0, \ldots, 4$ and

$$
\int^{\infty} \frac{1}{q_{i}(s)} \mathrm{d} s=\infty, \quad i=1,2,3 .
$$

However, the computation using the Lemmas 1 and 2 from [2] leading to canonical representation is very complicated and does not provide closed formulas for $q_{i}(t)$. This brings us to the question whether is it possible to establish a closed form formulas for $q_{i}$. The aim of this paper is to positively answer to this question, showing simultaneously the advantage of the result in the investigation of oscillatory properties of strongly noncanonical equations.

\section{Main results}

Throughout the paper we assume that (1.2) hold and so we can employ the notation

$$
\pi_{i}(t)=\int_{t}^{\infty} \frac{1}{r_{i}(s)} \mathrm{d} s, \quad \pi_{i j}(t)=\int_{t}^{\infty} \frac{1}{r_{i}(s)} \pi_{j}(s) \mathrm{d} s
$$

and

$$
\pi_{i j k}(t)=\int_{t}^{\infty} \frac{1}{r_{i}(s)} \pi_{j k}(s) \mathrm{d} s,
$$

where $i, j, k \in\{1,2,3\}$ are mutually different.

We start with the following auxiliary results which are elementary but very useful.

Lemma 1. Let (1.2) hold. Then

$$
\pi_{i j}(t)+\pi_{j i}(t)=\pi_{i}(t) \pi_{j}(t) .
$$

Proof. Since

$$
\left(\pi_{i}(t) \pi_{j}(t)\right)^{\prime}=-\frac{\pi_{j}(t)}{r_{i}(t)}-\frac{\pi_{i}(t)}{r_{j}(t)},
$$

an integration of this equality from $t$ to $\infty$, yields

$$
\pi_{i}(t) \pi_{j}(t)=\int_{t}^{\infty} \frac{1}{r_{i}(s)} \pi_{j}(s) \mathrm{d} s+\int_{t}^{\infty} \frac{1}{r_{j}(s)} \pi_{i}(s) \mathrm{d} s=\pi_{i j}(t)+\pi_{j i}(t) .
$$


To simplify our notation, we denote

$$
\Omega(t)=\pi_{12}(t) \pi_{23}(t)-\pi_{2}(t) \pi_{123}(t) .
$$

The following result provides an alternative formula for $\Omega(t)$.

Lemma 2. Let (1.2) hold. Then

$$
\pi_{12}(t) \pi_{23}(t)-\pi_{2}(t) \pi_{123}(t)=\pi_{21}(t) \pi_{32}(t)-\pi_{2}(t) \pi_{321}(t) .
$$

Proof. Proof of this lemma is similar to that of Lemma 1 and so it can be omitted.

Now, we are prepared to introduce the main result.

Theorem 1. The strongly noncanonical operator $\mathcal{L} y$ has the following unique canonical representation

$$
\mathcal{L} y=\frac{1}{\pi_{321}(t)}\left(\frac{r_{3} \pi_{321}^{2}}{\Omega}\left(\frac{r_{2} \Omega^{2}}{\pi_{321} \pi_{123}}\left(\frac{r_{1} \pi_{123}^{2}}{\Omega}\left(\frac{y}{\pi_{123}}\right)^{\prime}\right)^{\prime}\right)^{\prime}\right)^{\prime}(t) .
$$

Proof. Straightforward evaluation of $\mathcal{L} y$, with $\mathcal{L}$ as defined by (2.2) yields

$$
\mathcal{L}_{1} y=\frac{r_{1} \pi_{123}^{2}}{\Omega}\left(\frac{y}{\pi_{123}}\right)^{\prime}=\frac{r_{1} y^{\prime} \pi_{123}+y \pi_{23}}{\Omega} .
$$

Employing (2.1), we see that

$$
\begin{aligned}
\mathcal{L}_{2} y & =\frac{\Omega^{2}}{r_{2} \pi_{321} \pi_{123}}\left(\mathcal{L}_{1} y\right)^{\prime} \\
& =\frac{r_{2}\left(r_{1} y^{\prime}\right)^{\prime} \Omega-r_{1} y^{\prime}\left(\pi_{321}-\pi_{1} \pi_{32}\right)+y \pi_{32}}{\pi_{321}} .
\end{aligned}
$$

It follows from Lemma 2 that $\pi_{12}(t)+\pi_{21}(t)=\pi_{1}(t) \pi_{2}(t)$ and so

$$
\left(\mathcal{L}_{2} y\right)^{\prime}=\frac{\left(r_{2}\left(r_{1} y^{\prime}\right)^{\prime}\right)^{\prime} \Omega-r_{1} y^{\prime} \frac{1}{r_{3}} \pi_{12}-y \frac{1}{r_{3}} \pi_{2}}{\pi_{321}}+\frac{r_{2}\left(r_{1} y^{\prime}\right)^{\prime} \Omega \pi_{21}+r_{1} y^{\prime}\left(\pi_{1} \pi_{32} \pi_{21}-\pi_{321} \pi_{21}\right)+y \pi_{32} \pi_{21}}{r_{3} \pi_{321}^{2}}
$$

On the other hand, by Lemma 1 and 2

$$
\begin{aligned}
r_{1} y^{\prime}\left(\pi_{1} \pi_{32} \pi_{21}-\pi_{321} \pi_{21}-\pi_{12} \pi_{321}\right) & =r_{1} y^{\prime}\left(\pi_{1} \pi_{32} \pi_{21}-\pi_{321} \pi_{1} \pi_{2}\right) \\
& =r_{1} y^{\prime} \pi_{1} \Omega .
\end{aligned}
$$

Consequently,

$$
\left(\mathcal{L}_{2} y\right)^{\prime}=\frac{\left(r_{2}\left(r_{1} y^{\prime}\right)^{\prime}\right)^{\prime} \Omega}{\pi_{321}}+\frac{r_{2}\left(r_{1} y^{\prime}\right)^{\prime} \Omega \pi_{21}+r_{1} y^{\prime} \pi_{1} \Omega+y \Omega}{r_{3} \pi_{321}^{2}} .
$$

Then

$$
\mathcal{L}_{3} y=\frac{r_{3} \pi_{321}^{2}}{\Omega}\left(\mathcal{L}_{2} y\right)^{\prime} s=r_{3}\left(r_{2}\left(r_{1} y^{\prime}\right)^{\prime}\right)^{\prime} \pi_{321}+r_{2}\left(r_{1} y^{\prime}\right)^{\prime} \pi_{21}+r_{1} y^{\prime} \pi_{1}+y
$$

Finally

$$
\frac{1}{\pi_{321}(t)}\left(\mathcal{L}_{3} y\right)^{\prime}=\left(r_{3}(t)\left(r_{2}(t)\left(r_{1}(t) y^{\prime}(t)\right)^{\prime}\right)^{\prime}\right)^{\prime}=\mathcal{L} y
$$

which means that the operators (1.1) and (2.2) are equivalent. 
Now we shall show that operator (2.2) is canonical. Direct computation shows that

$$
\int_{t_{0}}^{t} \frac{1}{q_{1}(s)} \mathrm{d} s=\int_{t_{0}}^{t} \frac{\Omega(s)}{r_{1}(s) \pi_{123}^{2}(s)} \mathrm{d} s=\int_{t_{0}}^{t}\left(\frac{\pi_{12}(s)}{\pi_{123}(s)}\right)^{\prime} \mathrm{d} s=\frac{\pi_{12}(t)}{\pi_{123}(t)}-c_{1} .
$$

Applying twice the L'Hospital rule, we get

and so

$$
\lim _{t \rightarrow \infty} \frac{\pi_{12}(t)}{\pi_{123}(t)}=\lim _{t \rightarrow \infty} \frac{1}{\pi_{3}(t)}=\infty
$$

Similarly

$$
\int_{t_{0}}^{t} \frac{1}{q_{3}(s)} \mathrm{d} s=\int_{t_{0}}^{t} \frac{\Omega(s)}{r_{3}(s) \pi_{321}^{2}(s)} \mathrm{d} s=\left(\frac{\pi_{32}(t)}{\pi_{321}(t)}-c_{3}\right) \rightarrow \infty \text { as } t \rightarrow \infty .
$$

To evaluate the last one integral it is useful to see that

$$
\begin{aligned}
\left(\frac{\pi_{321}(t) \pi_{23}(t)}{\pi_{32}(t) \Omega(t)}+\frac{\pi_{3}(t)}{\pi_{32}(t)}\right)^{\prime} & =\left(\frac{\pi_{3}(t) \pi_{21}(t)-\pi_{321}(t)}{\Omega(t)}\right)^{\prime} \\
& =\frac{\pi_{321}(t) \pi_{123}(t)}{r_{2}(t) \Omega^{2}(t)}
\end{aligned}
$$

Therefore,

$$
\begin{aligned}
\int_{t_{0}}^{t} \frac{1}{q_{2}(s)} \mathrm{d} s & =\int_{t_{0}}^{t} \frac{\pi_{321}(s) \pi_{123}(s)}{r_{2}(s) \Omega^{2}(s)} \mathrm{d} s=\int_{t_{0}}^{t}\left(\frac{\pi_{321}(s) \pi_{23}(s)}{\pi_{32}(s) \Omega(s)}+\frac{\pi_{3}(s)}{\pi_{32}(s)}\right)^{\prime} \mathrm{d} s \\
& =\frac{\pi_{321}(t) \pi_{23}(t)}{\pi_{32}(t) \Omega(t)}+\frac{\pi_{3}(t)}{\pi_{32}(t)}-c_{2} .
\end{aligned}
$$

Moreover,

$$
\lim _{t \rightarrow \infty} \frac{\pi_{3}(t)}{\pi_{32}(t)}=\lim _{t \rightarrow \infty} \frac{1}{\pi_{2}(t)}=\infty
$$

and we conclude that the operator (2.2) is canonical. By Trench's result [2] there exists the only one canonical representation of $\mathcal{L}$ (up to multiplicative constants with product 1 ) and so our canonical form is unique.

We support our results with couple of illustrative examples.

Example 1. Let us consider the following operator

$$
\mathcal{L} y=\left(t^{\gamma}\left(t^{\beta}\left(t^{\alpha} y^{\prime}(t)\right)^{\prime}\right)^{\prime}\right)^{\prime}, \quad \alpha>1, \quad \beta>1, \quad \gamma>1 .
$$

By Theorem 1, this operator can be rewritten in canonical form as

$$
\mathcal{L} y=\frac{1}{t^{3-\alpha-\beta-\gamma}}\left(t^{2-\alpha}\left(t^{2-\beta}\left(t^{2-\gamma}\left(\frac{y(t)}{t^{3-\alpha-\beta-\gamma}}\right)\right)^{\prime}\right)^{\prime}\right)^{\prime}
$$

Example 2. The operator

$$
\mathcal{L} y=\left(\mathrm{e}^{\gamma t}\left(\mathrm{e}^{\beta t}\left(\mathrm{e}^{\alpha t} y^{\prime}(t)\right)^{\prime}\right)^{\prime}\right)^{\prime}, \quad \alpha>0, \quad \beta>0, \quad \gamma>0 .
$$

can be represented in canonical form as

$$
\mathcal{L} y=\mathrm{e}^{(\alpha+\beta+\gamma) t}\left(\mathrm{e}^{-\alpha t}\left(\mathrm{e}^{-\beta t}\left(\mathrm{e}^{-\gamma t}\left(\mathrm{e}^{(\alpha+\beta+\gamma) t} y(t)\right)^{\prime}\right)^{\prime}\right)^{\prime}\right)^{\prime} .
$$




\section{Applications to differential equations}

Theorem 1 can be applied to study oscillatory properties of differential equations. We are going to outline one such application based on comparison principle.

Corollary 1. Noncanonical differential equation $(E)$ can be written in canonical form as

$$
\frac{1}{\pi_{321}(t)}\left(\frac{r_{3} \pi_{321}^{2}}{\Omega}\left(\frac{r_{2} \Omega^{2}}{\pi_{321} \pi_{123}}\left(\frac{r_{1} \pi_{123}^{2}}{\Omega}\left(\frac{y}{\pi_{123}}\right)^{\prime}\right)^{\prime}\right)^{\prime}\right)^{\prime}(t)+p(t) y(\tau(t))=0 .
$$

Setting $z(t)=y(t) / \pi_{123}(t)$ we get the following comparison result that reduces oscillation of strongly noncanonical equation to that of canonical equation.

Corollary 2. Noncanonical equation (E) is oscillatory if and only if the canonical equation

$$
\left(\frac{r_{3} \pi_{321}^{2}}{\Omega}\left(\frac{r_{2} \Omega^{2}}{\pi_{321} \pi_{123}}\left(\frac{r_{1} \pi_{123}^{2}}{\Omega} z^{\prime}\right)^{\prime}\right)^{\prime}\right)^{\prime}(t)+\pi_{321}(t) \pi_{123}(\tau(t)) p(t) z(\tau(t))=0 .
$$

is oscillatory.

Example 3. Let us consider the fourth order differential equation

$$
\left(t^{\gamma}\left(t^{\beta}\left(t^{\alpha} y^{\prime}(t)\right)^{\prime}\right)^{\prime}\right)^{\prime}+p(t) y(\tau(t))=0
$$

with $\alpha>1, \beta>1, \gamma>1$. By Corollary 2, this equation is oscillatory if and only if the canonical equation

$$
\left(t^{2-\alpha}\left(t^{2-\beta}\left(t^{2-\gamma} z^{\prime}(t)\right)^{\prime}\right)^{\prime}\right)^{\prime}+(t \tau(t))^{3-\alpha-\beta-\gamma} p(t) z(\tau(t))=0
$$

is oscillatory. It is more convenient to study oscillation of (3.12) instead of (3.1).

Now, we are ready to study the properties of $(E)$ with the help of $\left(E_{c}\right)$. Without loss of generality, we can consider only with the positive solutions of $\left(E_{c}\right)$. The following result is a modification of Kiguradze's lemma [3].

Let us denote

$$
q_{1}(t)=\frac{r_{1}(t) \pi_{123}^{2}(t)}{\Omega(t)}, \quad q_{2}(t)=\frac{r_{2}(t) \Omega^{2}(t)}{\pi_{321}(t) \pi_{123}(t)}, \quad q_{3}(t)=\frac{r_{3}(t) \pi_{321}^{2}(t)}{\Omega(t)}
$$

and

$$
P(t)=\pi_{321}(t) \pi_{123}(\tau(t)) p(t) .
$$

Then $\left(E_{c}\right)$ can be rewritten as

$$
\left(q_{3}\left(q_{2}\left(q_{1} z^{\prime}\right)^{\prime}\right)^{\prime}\right)^{\prime}(t)+P(t) z(\tau(t))=0 .
$$

Lemma 3. Assume that $z(t)$ is an eventually positive solution of $\left(E_{c}\right)$, then either

$$
z(t) \in \mathcal{N}_{1} \Longleftrightarrow z^{\prime}(t)>0, \quad\left(q_{1} z^{\prime}\right)^{\prime}(t)<0, \quad\left(q_{2}\left(q_{1} z^{\prime}\right)^{\prime}\right)^{\prime}(t)>0
$$

or

$$
z(t) \in \mathcal{N}_{3} \Longleftrightarrow z^{\prime}(t)>0, \quad\left(q_{1} z^{\prime}\right)^{\prime}(t)>0, \quad\left(q_{2}\left(q_{1} z^{\prime}\right)^{\prime}\right)^{\prime}(t)>0
$$


Consequently, the set $\mathcal{N}$ of all positive solutions of $(E)$ has the decomposition

$$
\mathcal{N}=\mathcal{N}_{1} \cup \mathcal{N}_{3}
$$

To obtain oscillation of studied equation $(E)$, we need to eliminate both cases of possible non-oscillatory solutions.

Let us denote

$$
Q_{1}(t)=\left(\frac{1}{q_{2}(t)} \int_{t}^{\infty} \frac{1}{q_{3}(u)} \int_{u}^{\infty} P(s) \mathrm{d} s \mathrm{~d} u\right) \int_{t_{1}}^{\tau(t)} \frac{1}{q_{1}(u)} \mathrm{d} u
$$

and

$$
Q_{2}(t)=P(t) \int_{t_{1}}^{t} \frac{1}{q_{1}\left(s_{1}\right)} \int_{t_{1}}^{s_{1}} \frac{1}{q_{2}(u)} \int_{t_{1}}^{s} \frac{1}{q_{3}(s)} \mathrm{d} s \mathrm{~d} u \mathrm{~d} s_{1} .
$$

Theorem 2. Let (1.2) hold. Assume that both first-order delay differential equations

$$
x^{\prime}(t)+Q_{1}(t) x(\tau(t))=0
$$

and

$$
x^{\prime}(t)+Q_{2}(t) x(\tau(t))=0 .
$$

are oscillatory. Then $(E)$ is oscillatory.

Proof. Assume that $y(t)$ is an eventually positive solution of $(E)$, say for $t \geq t_{1}$. Then by Corollary $2, z(t)=$ $y(t) / \pi_{123}(t)$ is a solution of $\left(E_{c}\right)$.

It follows from Lemma 3 that either $z(t) \in \mathcal{N}_{1}$ or $z(t) \in \mathcal{N}_{3}$. At first, we admit that $z(t) \in \mathcal{N}_{1}$. Noting that $q_{1}(t) z^{\prime}(t)$ is decreasing, we see that

$$
z(t) \geq \int_{t_{1}}^{t} \frac{1}{q_{1}(u)} q_{1}(u) z^{\prime}(u) d u \geq q_{1}(t) z^{\prime}(t) \int_{t_{1}}^{t} \frac{1}{q_{1}(u)} d u .
$$

Integrating $\left(E_{c}\right)$ from $t$ to $\infty$, we have

$$
q_{3}\left(q_{2}\left(q_{1} z^{\prime}\right)^{\prime}\right)^{\prime}(t) \geq \int_{t}^{\infty} P(s) z(\tau(s)) \mathrm{d} s .
$$

Taking into account that $z(\tau(t))$ is increasing, the last inequality yields

$$
\left(q_{2}\left(q_{1} z^{\prime}\right)^{\prime}\right)^{\prime}(t) \geq z(\tau(t)) \frac{1}{q_{3}(t)} \int_{t}^{\infty} P(s) \mathrm{d} s .
$$

Integrating once more, we are led to

$$
-\left(q_{1} z^{\prime}\right)^{\prime}(t) \geq z(\tau(t)) \frac{1}{q_{2}(t)} \int_{t}^{\infty} \frac{1}{q_{3}(u)} \int_{u}^{\infty} P(s) \mathrm{d} s \mathrm{~d} u .
$$

Combining the last inequality with (3.5), one gets

$$
\begin{aligned}
-\left(q_{1} z^{\prime}\right)^{\prime}(t) & \geq\left(z^{\prime}(\tau(t)) \frac{q_{1}(\tau(t))}{q_{2}(t)} \int_{t}^{\infty} \frac{1}{q_{3}(u)} \int_{u}^{\infty} P(s) \mathrm{d} s \mathrm{~d} u\right) \int_{t_{1}}^{\tau(t)} \frac{1}{q_{1}(u)} \mathrm{d} u \\
& =q_{1}(\tau(t)) z^{\prime}\left(\tau(t) Q_{1}(t) .\right.
\end{aligned}
$$


Thus, the function $x(t)=q_{1}(t) z^{\prime}(t)$ is a positive solution of the differential inequality

$$
x^{\prime}(t)+Q_{1}(t) x(\tau(t)) \leq 0 .
$$

Hence, by Philos theorem [4], we conclude that the corresponding differential equation (3.3) also has a positive solution, which contradicts the assumptions of the theorem.

Now, we shall assume that $z(t) \in \mathcal{N}_{3}$. Since $q_{3}\left(q_{2}\left(q_{1} z^{\prime}\right)^{\prime}\right)^{\prime}$ is decreasing, we are led to

$$
\begin{aligned}
q_{2}\left(q_{1} z^{\prime}\right)^{\prime}(t) & \geq \int_{t_{1}}^{t} \frac{1}{q_{3}(s)} q_{3}(s)\left(q_{2}\left(q_{1} z^{\prime}\right)^{\prime}\right)^{\prime}(s) \mathrm{d} s \\
& \geq q_{3}\left(q_{2}\left(q_{1} z^{\prime}\right)^{\prime}\right)^{\prime}(t) \int_{t_{1}}^{t} \frac{1}{q_{3}(s)} \mathrm{d} s .
\end{aligned}
$$

Integrating the above inequality, one can verify that

$$
z^{\prime}(t) \geq q_{3}\left(q_{2}\left(q_{1} z^{\prime}\right)^{\prime}\right)^{\prime}(t) \frac{1}{q_{1}(t)} \int_{t_{1}}^{t} \frac{1}{q_{2}(u)} \int_{t_{1}}^{s} \frac{1}{q_{3}(s)} \mathrm{d} s \mathrm{~d} u
$$

Integrating once more, we see that $x(t)=q_{3}\left(q_{2}\left(q_{1} z^{\prime}\right)^{\prime}\right)^{\prime}(t)$ satisfies

$$
z(t) \geq x(t) \int_{t_{1}}^{t} \frac{1}{q_{1}\left(s_{1}\right)} \int_{t_{1}}^{s_{1}} \frac{1}{q_{2}(u)} \int_{t_{1}}^{s} \frac{1}{q_{3}(s)} \mathrm{d} s \mathrm{~d} u \mathrm{~d} s_{1} .
$$

Setting the last estimate into $\left(E_{c}\right)$, we see that $x(t)$ is a positive solution of the differential inequality

$$
x^{\prime}(t)+Q_{2}(t) x(\tau(t)) \leq 0,
$$

which in view of Philos theorem in [4] guarantees that the corresponding differential equation (3.4) has also a positive solution. This is a contradiction and the proof is complete now.

Applying suitable criteria for oscillation of (3.3) and (3.4), we immediately obtain the criteria for oscillation of (E). We use the one which is due to Ladde et al. [5].

Corollary 3. Let (1.2) hold. Assume that for $i=1,2$

$$
\liminf _{t \rightarrow \infty} \int_{\tau(t)}^{t} Q_{i}(s) \mathrm{d} s>\frac{1}{\mathrm{e}}
$$

hold. Then (E) is oscillatory.

We support our results by another example.

Example 4. Let us consider the general Euler delay differential equation

$$
\left(t^{\gamma}\left(t^{\beta}\left(t^{\alpha} y^{\prime}(t)\right)^{\prime}\right)^{\prime}\right)^{\prime}+a t^{\alpha+\beta+\gamma-4} y(\lambda t)=0
$$

with $\alpha>1, \beta>1, \gamma>1, a>0$, and $0<\lambda<1$. By Corollary 2 and Example 3, this equation is oscillatory if and only if the canonical equation

$$
\left(t^{2-\alpha}\left(t^{2-\beta}\left(t^{2-\gamma} z^{\prime}(t)\right)^{\prime}\right)^{\prime}\right)^{\prime}+a \lambda^{\alpha+\beta+\gamma-3} t^{\alpha+\beta+\gamma-4} z(\lambda t)=0
$$


is oscillatory. The straightforward computation yields that

$$
Q_{1}(t) \sim \frac{a \lambda^{2-\alpha-\beta}}{(\alpha+\beta+\gamma-3)(\beta+\gamma-2)(\gamma-1)} t^{-1}
$$

and

$$
Q_{2}(t) \sim \frac{a \lambda^{3-\alpha-\beta-\gamma}}{(\alpha+\beta+\gamma-3)(\alpha+\beta-2)(\alpha-1)} t^{-1} .
$$

By Corollary 4 considered equation is oscillatory, provided that both conditions

$$
\frac{a \lambda^{2-\alpha-\beta}}{(\alpha+\beta+\gamma-3)(\beta+\gamma-2)(\gamma-1)} \ln \left(\frac{1}{\lambda}\right)>\frac{1}{\mathrm{e}}
$$

and

$$
\frac{a \lambda^{3-\alpha-\beta-\gamma}}{(\alpha+\beta+\gamma-3)(\alpha+\beta-2)(\alpha-1)} \ln \left(\frac{1}{\lambda}\right)>\frac{1}{\mathrm{e}}
$$

are satisfied.

\section{Summary}

In this paper we provided canonical representation for strongly noncanonical operator. This canonical transformation is easy and immediate. Moreover, we point out its application in the oscillation theory.

Acknowledgement: The paper has been supported by the grant project KEGA 035TUKE-4/2017.

\section{References}

[1] Bartusek M., Dosla Z., Asymptotic problems for fourth-order nonlinear differential equations. Boundary Value Problems 2013, 1-15

[2] Trench W., Canonical forms and principal systems for general disconjugate equations, Trans. Amer. Math. Soc. 184, 1974, 319-327

[3] Kiguradze I. T., Chanturia T. A., Asymptotic Properties of Solutions of Nonautonomous Ordinary Differential Equation, Kluwer Acad. Publ., Dordrecht 1993

[4] Philos Ch. G., Ot the existence of nonoscillatory solutions tending to zero at $\infty$ for differential equations with positive delay, Arch. Math., 36, 1981, 168-178

[5] Ladde G. S., Lakshmikantham V., Zhang B. G., Oscillation Theory of Differential Equations with Deviating Argument, Marcel Dekker, New York, 1987

[6] Dzurina J., Comparison theorems for nonlinear ODE's, Math. Slovaca 42, 199), 299-315

[7] Dzurina J., Kotorova R., Zero points of the solutions of a differential equation, Acta Electrotechnica et Informatica 7, 2007, 26-29

[8] Jadlovska, I.,Application of Lambert W function in oscillation theory, Acta Electrotechnica et Informatica 14, 2014, 9-17

[9] Koplatadze R., Kvinkadze G., Stavroulakis I. P., Properties A and B of $\mathrm{n}$-th order linear differential equations with deviating argument, Georgian Math. J. 6, 1999, 553-566

[10] Kitamura Y., Kusano T., Oscillations of first-order nonlinear differential equations with deviating arguments, Proc. Amer. Math. Soc., 78, 1980, 64-68

[11] Kusano T., Naito M., Comparison theorems for functional differential equations with deviating arguments, J. Math. Soc. Japan 3, 1981, 509-533

[12] Zhang T. Li, Ch., Thandapani E., Asymptotic behavior of fourth-order neutral dynamic equations with noncanonical operators, Taiwanese J. Math. 18, 2014, 1003-1019

[13] Mahfoud W. E., Comparison theorems for delay differential equations, Pacific J. Math. 83, 1979, No. 83, 187-197 